stakeholders such as occupational health and safety professionals (HSPs) on injury risk factors during hot weather may assist in informing injury prevention efforts.

Methods A national online survey of HSPs was undertaken. Collected data included perspectives on injury experiences, current preventive measures, training, policies and guidelines, and barriers for prevention. Results were analyzed descriptively and a log-poisson regression was used to identify risk factors associated with reported injuries during hot weather at workplaces the HSPs visited/managed.

Results In total there were 307 respondents, the majority of who acknowledged the potential for increased risk of occupational injuries in hot weather. A variety of injury types and mechanisms were reported, including manual handling injuries, hand injuries, wounds or lacerations and loss of control of power tools.

Work factors significantly associated with reported injuries included problems with personal protective equipment; lack of shade; inadequate hydration and rest breaks, and problems with supervision.

Less than half (42\%) of HSPs stated the availability of adequate heat training for staff and 54\% reported the provision for outdoor work to cease if temperatures are extreme as a preventive measure in workplaces they visited/managed. HSPs recommended 'heat stress training' as the most important preventive measure for outdoor and indoor workers. Reported barriers for prevention include: lack of awareness by workers and supervisors of injury risks, and management concerns about productivity loss and/or deadlines.

Conclusion These findings point to a range of modifiable work and organisational risk factors for injury during hot weather. More attention to these factors, in conjunction with traditional interventions for heat-induced illness, could enhance injury prevention in the workplace.

\section{B.5 OCCUPATIONAL HEAT EXPOSURE AND CARDIOVASCULAR HEALTH RISKS RELATED TO CLIMATE CHANGE IN PACIFIC COUNTRIES}

${ }^{1}$ Ashley Akerman*, ${ }^{1} \mathrm{Jim}$ Cotter, ${ }^{2}$ Tord Kjellstrom. ${ }^{1}$ School of Physical Education, Sport and Exercise Sciences; University Of Otago, Dunedin, New Zealand; ${ }^{2}$ National Centre for Epidemiology and Population Health; Australian National University, Canberra, Australia

\subsection{6/OEM-2019-EPI.196}

Recent global analysis of current and future ambient heat conditions has shown that occupational heat exposure in Pacific countries, measured or projected as WBGT, is among the most extreme in the world. Many Pacific countries face a serious and unprecedented collision of adverse environmental, sociocultural/demographic and chronic health factors. Environmentally, high temperatures and humidity exist several months each year, reducing physical work capacity. Conservative assessments reveal annual loss of healthy work hours for a person working at $400 \mathrm{~W}$ metabolic rate in countries like Papua New Guinea, Kiribati, Nauru and Guam increasing from $2 \%-3 \%$ to $9 \%-18 \%$ by 2100 , based on the RCP6.0 pathway for climate change. Socio-culturally, such countries have low average income levels and high reliance on primary industries, so occupational and domestic exposure to ambient heat is chronic and somewhat intractable. Health wise, such countries have among the highest burden of chronic cardiometabolic disease in the world. Diabetes is already prevalent $(20 \%-35 \%)$, as is hypertension. Casual links between heat exposure and cardiovascular health problems is strengthening and these health problems are becoming a major threat to health in Pacific countries. A diet that is increasingly more divergent from traditional foods is one key factor, but increasing heat conditions will contribute, particularly for people working in physically intensive occupations. Obesity is also prevalent, which is relevant because it adds to heat production while concurrently reducing heat loss, thereby impairing work capacity and tolerance of humid heat. Collectively these factors compromise thermal tolerance, reduce functional capacity and contribute to cardiometabolic disease. This paper will analyse the physiological base for the heat effects on cardiovascular disease with particular reference to occupational heat exposure, and estimate the impacts of climate change in the Pacific countries as well as identify solutions to reduce the future impacts.

\section{B.6 OCCUPATIONAL HEAT STRESS DUE TO CLIMATE CHANGE: ESTIMATING FUTURE HEAT WAVE HAZARDS}

${ }^{1,2}$ Matthias Otto* 2,3 Tord Kjellstrom, 1,2 ${ }^{\text {Bruno Lemke. }}{ }^{1}$ Nelson Marlborough Institute of Technology (NMIT), Nelson, New Zealand; ${ }^{2}$ Climate Heat Impact Research Programme (CHIRP), Ruby Bay, New Zealand; ${ }^{3}$ Australian National University (ANU), Canberra, Australia

\subsection{6/OEM-2019-EPI.197}

Exposure to extreme heat negatively affects occupational health. Heat stress indices like Wet Bulb Globe Temperature (WBGT) combine temperature and humidity and allow quantifying the climatic impact on human physiology and clinical health. Multi-day periods of high heat stress (aka. heat waves) affect occupational health and productivity independently from the absolute temperature levels; e.g. well-documented heatwaves in Europe caused disruption, hospitalisations and deaths (2003 French heat wave: more than 1000 extra deaths, 15-65 years, mainly men) even though the temperatures were within the normal range of hotter countries.

Climate change is likely to increase frequency and severity of periods of high heat stress. However, current global gridcell based climate models are not designed to predict heat waves, neither in terms of severity or frequency.

By analysing 37 years of historic daily heat index data from almost 5000 global weather stations and comparing them to widely used grid-cell based climate model outputs over the same period, our research explores methods to assess the frequency and intensity of heat waves as well as the associated occupational health effects at any location around the world in the future.

Weather station temperature extreme values (WBGT) for the 3 hottest days in 30 years exceed the mean WBGT of the hottest month calculated from climate models in the same grid-cell by about 2 degrees in the tropics but by 10 degrees at higher latitudes in temperate climate regions.

Our model based on the relationship between actual recorded periods of elevated heat-stress and grid-cell based climate projections, in combination with population and employment projections, can quantify national and regional productivity loss and health effects with greater certainty than is currently the case. 